\title{
A Case Report of Giant Cell Tumor of Proximal Fibula in Children
}

\author{
Sushil Adhikari ${ }^{1}$, Arun Sigdel ${ }^{1}$, Rajesh Kumar Sah², Luna Devkota ${ }^{3}$ \\ ${ }^{1}$ Orthopedic Surgery Unit, Department of Surgical Oncology, B.P. Koirala Memorial Cancer Hospital Chitwan, Nepal \\ ${ }^{2}$ Department of Orthopedics, College of Medical Sciences Teaching Hospital Chitwan, Nepal \\ ${ }^{3}$ Department of Public Health, Shree Medical and Technical College, Chitwan,Nepal
}

\begin{abstract}
Giant cell tumour (GCT) is histopathologically benign tumor of long bone particularly in distal femur and the proximal tibia. It commonly occurs in adults of age $20-40$ years but rare in children. GCT is considered to be locally aggressive tumor and tendency of recurrence is higher even after surgery.

The clinical features are nonspecific, the principle symptoms are pain, swelling and limiting adjacent joint movements. Diagnosis is based on the radiographic appearance and histopathological findings .In our case X-ray showed ill defined lytic lesion on proximal fibula with cortical thinning and MRI finding revealed expansile lytic lesion in meta-epiphysis of right fibula $16 \times 16 \times 28 \mathrm{~mm}$ adjacent to growth plate with fluid level. The sclerotic rim appears hypointense on T1 \& hyperintense on T2. Core needle biopsy showed giant cell tumor on proximal fibula. Considering the risk of recurrence wide local excision was done. Management of GCT of proximal fibula in young patient is critical for preventing recurrence and enhancing functional outcomes by saving adjacent anatomical structure. No evidence of local recurrence and metastasis was found in 24 months of follow up.
\end{abstract}

Keywords: Giant Cell Tumor, Wide Local Excision, Fibula.

\section{Introduction}

Histopathologically Giant cell tumor (GCT) is a common benign tumor of long bone. It is classified as osteoclastic gaint cell rich tumors of intermediate grade with local aggressiveness and capacity to metastasize. GCT commonly occurs in mature skeleton. The incidence of occurrence lies between age 20 to 40 years but rare in children. ${ }^{1-3}$ The common sites for GCT in long bone are distal femur and proximal tibia. ${ }^{4-5}$ Literatures showed that GCT in the immature skeleton varies from $1.8 \%$ to $10.6 \% .6 \mathrm{~A}$ retrospective study conducted in GCT by Strom et.al in Oslo, Norway from 1984-2015 found that out of 154 patients $89 \%$ were more than 15 years. This finding reveled that GCT is more in mature skeleton than in immature skeleton. The biological characteristics, symptom and treatment of the tumor in children do not differ from the adults. ${ }^{7-8}$ The principal symptoms are pain and swelling around affected joints followed by limited movements and sometimes presents with pathological fractures. The definitive diagnosis is based on radiographic and histopathological finding. Universally surgery is primary treatment for GCT. Commonly practicing surgical treatment are extended currattage with through the use of power burr, Phenol, liquid nitrogen, bone cement, hydrogen peroxide, zinc chloride and argon beam cauterization. Selection of appropriate surgical treatments plays a vital role in reducing recurrence rate for functional as well as oncological outcomes.

\section{Case report}

Seven years boy came to the BPKMCH with the features

\section{Correspondence}

Dr. Sushil Adhikari, Orthopedic Surgery Unit, Department of Surgical Oncology, BPKMCH Chtiwan, Nepal. drsushil6550@gmail.com 
of pain and swelling over lateral aspect of right proximal leg for two months duration. Pain was gradual in onset, dull aching, and progressive, localized without increasing on knee movements. There was no history of fever, limping, instability, locking and other constitutional symptoms. Examination showed diffused swelling over proximal fibula at lateral knee region (figure 1). X-ray showed ill defined lytic lesion on proximal metaphyseal region of fibula with cortical thinning (figure 2). For further confirmation of diagnosis MRI was done and finding revealed expansile lytic lesion in metaphysis of right fibula $16 \times 16 \times 28 \mathrm{~mm}$ adjacent to growth plate with fluid level. The sclerotic rim appears hypointense on T1 \& hyperintense on T2. Hematological and biochemical parameters were normal. Core needle biopsy was taken out and showed giant cell tumor. Considering recurrence risk patient was counseled for excision of fibular head instead of extended curettage. Wide excision of tumor was done protecting common peroneal nerve and lateral collateral ligaments and bicep femoris was anchored at proximal tibia with non absorbable sutures (figure 3, 4). Histopathological examination showed numerous multinucleated giant cells scattered among oval to spindle cells without atypia or mitotic features suggesting giant cell tumors. Post operatively child was kept on supportive brace for two weeks and gradually walking as pain tolerated. No ligament instability on varus stress test and can walk without pain and squats normally. There is no evidence of local recurrence \& metastasis till 24 months follow up (figure 5). The patient was kept for regular follow up for identifying local recurrence swiftly. Informed consent was taken from patient's parties for disseminating valuable information for exploring knowledge.

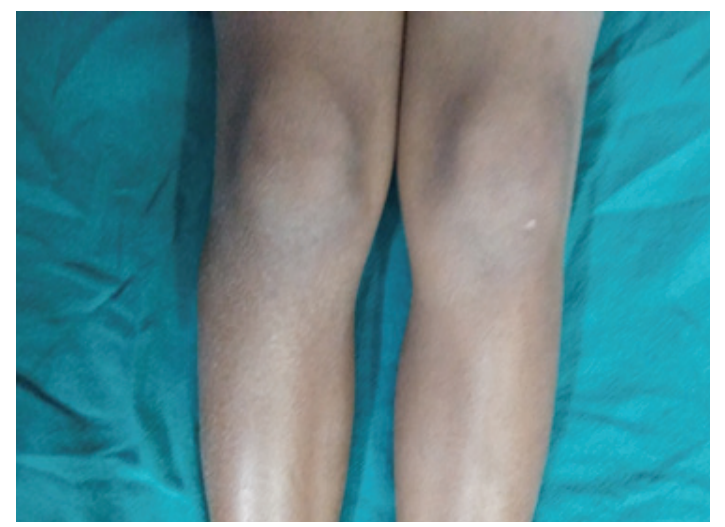

Figure 1 : Pre-operative clinical picture

\section{Discussion}

GCT is benign tumor with local aggressiveness thus it should be well treated. The incidence of occurrence is higher in adults between 20-40 years of age and more common sites are distal femur and proximal tibia. ${ }^{1-3}$ In our case, 7 years boy was diagnosed with GCT of proximal fibula. Studies suggested that both age and location of tumor is the rare condition..${ }^{9}{ }^{10}$ Being a rare disease limited studies has been conducted regarding GCT of children. Different studies showed GCT in immature skeleton representing $0.5 \%-10.6 \%$ of total GCT. ${ }^{11}$ Picci et al. have reported 6 cases of GCT in children around knee. ${ }^{12}$ GCT is locally aggressive tumor with higher tendency to recur after removal. ${ }^{13,14}$ The recurrence rate of tumor after surgery varies between studies. A multicentre retrospective study conducted in China between 2000-2014 regarding recurrence rate of primary GCT around knee among aged 20-39 years population was found $23.4 \%$ of local recurrence rate and according to location recurrence rate was also higher in proximal fibula as compared with distal femur. ${ }^{9}$

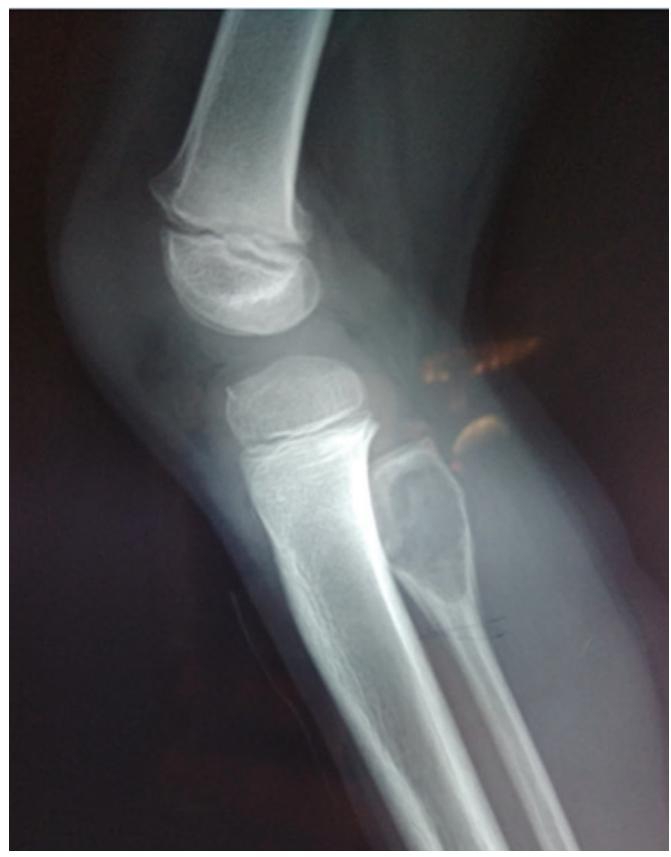

Figure 2 : Pre-op Xray knee

Selecting the appropriate treatment is the way to minimize the recurrence rate. But treatment dilemmas still existing related to functional and oncological outcomes. The aim of the treatment was to remove the tumor completely preserving surrounding anatomical structures for functional outcome. Treatment of GCT in children is 
same like in adults with similar rate of recurrence. ${ }^{7,8}$ we performed wide local excision of tumor saving common peroneal nerve and lateral collateral ligament and bicep femoris muscle to proximal tibia with non-absorbable sutures. The studies suggested that the rate of recurrence is lower with the wide local excision. ${ }^{10,13-16}$

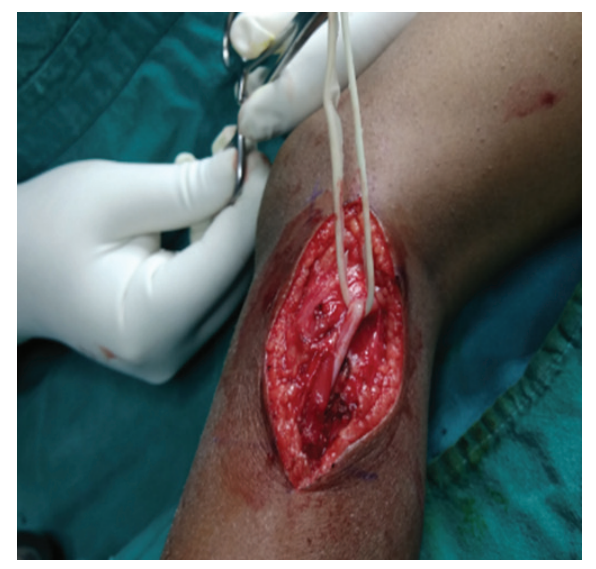

Figure 3 : Intra-operative picture showing common peroneal nerve superior to tumor bone.

A retrospective study conducted in China regarding recurrence rate of GCT around the knee showed higher rates of recurrence in treatment with intralesional curettage and curettage combined with resection as compared with en bloc marginal resection. ${ }^{9}$ A study conducted by Klenke et.al between 1958 -2005 found lower recurrence rate in patient treated with wide resection was only $5 \%$ and $25 \%$ with intralesional surgery. ${ }^{16}$ In wide local excision, preserving peroneal nerve is crucial for preventing functional impairment of the long bone.

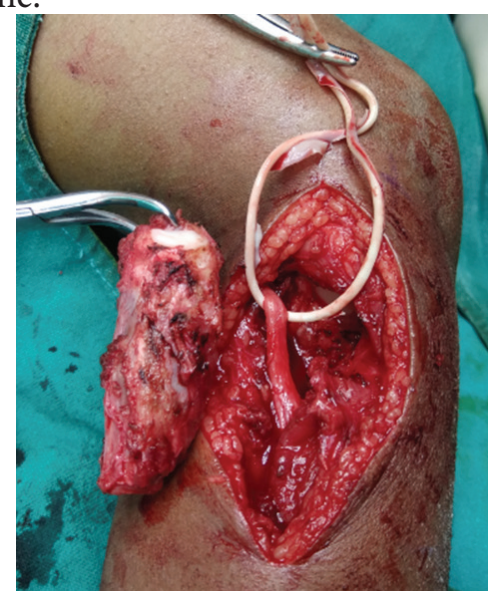

Figure 4 : Intr-operative picture showing excised tumor tissue with intact common peroneal nerve.

\section{Conclusion}

GCT is benign tumors of bone with local aggressiveness and higher tendency to recur after surgery. Considering aggressiveness of tumor wide local excision preserving common peroneal nerve and anchoring lateral collateral ligament was suitable option for optimum functional and oncological outcome. There was no evidence of local recurrence after 24 months of follow up.

GCT is rare condition in children in terms of age as well as site. The aim of this study was to explore knowledge on rare disease for contributing in the field of Orthopedic Oncology.

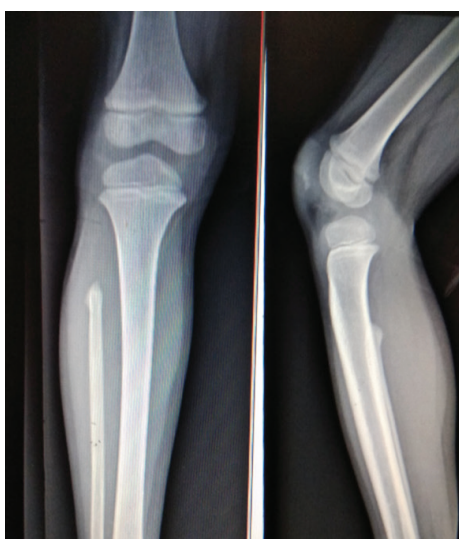

Figure 5 : 24 months follow up xray showing no feature of local recurrence.

\section{Conflict of Interest}

The authors declare that there is no conflict of interests regarding the publication of this paper.

\section{Acknowledgement}

We would like to acknowledge our patient for valuable information. We also like to include note of thanks to who are involved in care of this patient at BPKMCH.

\section{Reference}

1. Cheng DD, Hu T, Zhang HZ, Huang J, Yang QC. Factors affecting the recurrence of giant cell tumor of bone after surgery: a clinicopathological study of 80 cases from a single center. Cellular Physiology and Biochemistry. 2015;36(5):1961-70.

2. Dominic KP, Dijoe D, Aravind R. Extended curettage and reconstruction with proximal fibula 
for treating giant cell tumor of lateral femoral condyle: A prospective study. Archives of Clinical and Experimental Surgery (ACES). 2017;6(4):18994.

3. He H, Zeng H, Luo W, Liu Y, Zhang C, Liu Q. Surgical treatment options for giant cell tumors of bone around the knee joint: extended curettage or segmental resection?. Frontiers in oncology. 2019;9:946.

4. Sukpanichyingyong S, Sangkomkamhang T. Clinical Outcomes of Grade 3 Giant Cell Tumor of Bone After Extended Intralesional Curettage. J Orthop Oncol. 2017;3(118):2.

5. Hasan $\mathrm{O}$, Ali $\mathrm{M}$, Mustafa $M$, Ali A, Umer M. Treatment and recurrence of giant cell tumors of bone-A retrospective cohort from a developing country. Annals of Medicine and Surgery. 2019 Dec 1;48:29-34.

6. Puri A, Agarwal MG, Shah M, Jambhekar NA, Anchan C, Behle S. Giant cell tumor of bone in children and adolescents. Journal of Pediatric Orthopaedics. 2007 Sep 1;27(6):635-9.

7. Strøm TM, Skeie AT, Lobmaier IK, Zaikova O. Giant cell tumor: a rare condition in the immature skeleton-a retrospective study of symptoms, treatment, and outcome in 16 children. Sarcoma. 2016 Jan 1;2016.

8. Pandey S. Giant Cell Tumor Arising from Fibular Head in Immature Skeleton Treated with Marginal Excision. Journal of College of Medical SciencesNepal. 2018 Sep 30;14(3):170-3.

9. Hu P, Zhao L, Zhang H, Yu X, Wang Z, Ye Z, Wu S, Guo S, Zhang G, Wang J, Ning X. Recurrence rates and risk factors for primary giant cell tumors around the knee: a multicentre retrospective study in China. Scientific reports. 2016 Nov 9;6:36332.

10. Pawar E, Aher G, Gop A, Shah NZ, Ramteke U, Amin A, Kakde S. Management of giant cell tumor of fibular head with curettage: A case report. International Journal of Orthopaedics. 2019;5(1):63-6.

11. Unni KK, Inwards CY. Dahlin's bone tumors: general aspects and data on 10,165 cases. Lippincott Williams \& Wilkins; 2010.

12. Picci PI, Manfrini MA, Zucchi VI, Gherlinzoni FR, Rock MI, Bertoni FR, Neff JR. Giant-cell tumor of bone in skeletally immature patients. The Journal of bone and joint surgery. American volume. 1983 Apr;65(4):486-90.

13. Puri A, Agarwal M. Treatment of giant cell tumor of bone: Current concepts. Indian journal of orthopaedics. 2007 Apr;41(2):101.

14. van der Heijden L, Dijkstra PS, van de Sande MA, Kroep JR, Nout RA, van Rijswijk CS, Bovée JV, Hogendoorn PC, Gelderblom H. The clinical approach toward giant cell tumor of bone. The oncologist. 2014 May;19(5):550.

15. Inatani $\mathrm{H}$, Yamamoto $\mathrm{N}$, Hayashi $\mathrm{K}$, Kimura $\mathrm{H}$, Takeuchi A, Miwa S, Higuchi T, Abe K, Taniguchi Y, Yamada S, Okamoto H. Surgical management of proximal fibular tumors: A report of 12 cases. Journal of Bone Oncology. 2016 Nov 1;5(4):163-6.

16. Klenke FM, Wenger DE, Inwards CY, Rose PS, Sim FH. Giant cell tumor of bone: risk factors for recurrence. Clinical Orthopaedics and Related Research ${ }^{\oplus} 2011$ Feb 1;469(2):591-9. 